expression. They then performed literature searches and used RNA-silencing techniques to further narrow down their results.

The team focused on TMEM97 because it is highly expressed in sterol-depleted cells and is targeted by sterol-response-element binding proteins, which also activate other cholesterol regulatory genes. Knocking down its expression led to decreased cholesterol uptake and lowered cholesterol levels in cells. The authors suggest that their combined screening technique offers a powerful method for pinpointing genes in particular pathways.

\section{MICROBIOLOGY}

\section{Supershedding mice}

Infect. Immun. doi:10.1128/IAI.00558-09 (2009)

Clostridium difficile, a spore-forming anaerobic bacterium that inhabits the gut of as many as $5 \%$ of humans, is a leading cause of antibiotic-induced diarrhoea.

When a carrier takes antibiotics, these can wipe out other gut microbiota and send $C$. difficile into overdrive, which can lead to rampant spread of the bacterium in hospital

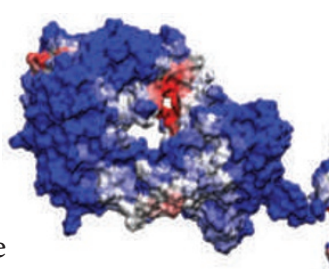
settings. Trevor Lawley of the Wellcome Trust Sanger Institute in Hinxton, UK, and his colleagues have developed a new mouse model for the condition.

Between two and three days after treatment with the antibiotic clindamycin, mice carrying $C$. difficile shed a millionfold more spores in their faeces than before. More than half of the mice retained this 'supershedder' status for several weeks. Uninfected mice housed with supershedders became carriers themselves; because the bacterial spores are ethanol resistant, cages had to be thoroughly cleaned with sporicides.

\section{GEOLOGY}

\section{Earth-shattering research}

Geology 37, 615-618 (2009)

How does a rock weather away into clay? Usually, it is helped along by fungi, in a process now examined in detail by Steeve Bonneville at the University of Leeds, UK, and his colleagues.

The team grew tree seedlings and a symbiotic fungus Paxillus involutus in an experimental set-up that included the mineral biotite, and followed the action with high-resolution microscopy. The fungus enhances the weathering of biotite, not by exuding chemically active substances as

was previously thought, but by physically disrupting the structure of the mineral with its hyphae. This is followed by chemical changes as the fungus removes potassium and oxidizes iron within the mineral. Soon, the rock is no more.

\title{
COMPUTATIONAL BIOLOGY
}

\section{Unstuck by design}

Proc. Natl Acad. Sci. USA doi:10.1073/

pnas.0904191106 (2009)

Computational predictions could make it easier to design biopharmaceuticals that don't stick together. Aggregation can destroy the effectiveness of antibody-based drugs or, worse, spur an unwanted immune response. But current methods to stabilize antibodies involve trial and error, which can be costly.

Bernhardt Trout of the Massachusetts Institute of Technology in Cambridge and his colleagues have developed a method to predict the exposed areas

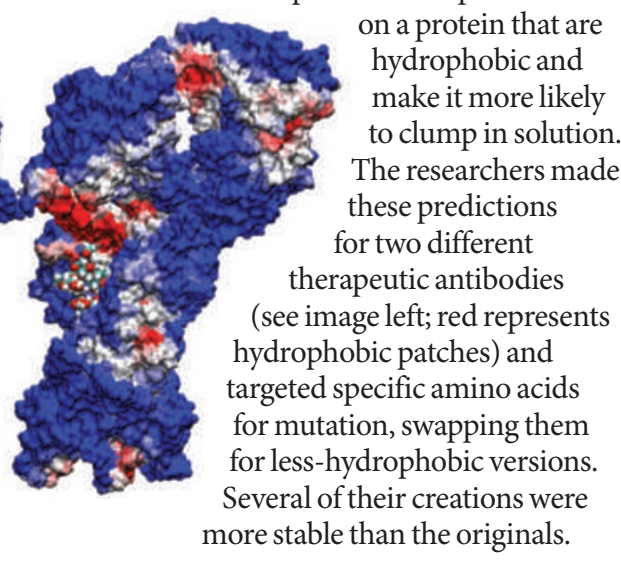

NEUROLOGY

\section{Feeling out autism}

Nature Neurosci. doi:10.1038/nn.2356 (2009) Children with autism spectrum disorder (ASD) may rely more on physical feedback than visual cues when performing tasks.

Reza Shadmehr at Johns Hopkins University in Baltimore, Maryland, and his colleagues asked children with ASD and children with typical development to play a video game in which they learn to use a robotic arm to capture animals. As training progresses, a force is applied to the arm that the children must correct for. In some instances, the corrective force that the children apply was measured, revealing how their brains build a generalized mental model of the way in which the robotic arm works.

Those children with ASD who were most reliant on the physical feedback to build this mental model had larger deficits in motor control, imitation and even social function.
JOURNAL CLUB

\author{
James J. Collins \\ Howard Hughes Medical \\ Institute, Boston University, \\ Massachusetts
}

\section{A bioengineer gets schooled by Escherichia coli.}

The ability to learn from situations and to predict certain outcomes sets us apart from many living things. It prevents many of us from chasing balls into busy streets or placing bottles of ethanol near Bunsen burners. Still, it didn't stop thousands of US researchers submitting applications for the National Institutes of Health's Challenge Grants - funded by President Barack Obama's economic stimulus package despite an expected success rate little better than one or two per cent.

To enjoy the benefits of learning and predictive behaviour, we usually assume that you need a nervous system or at least a neuron. So it was surprising to read that Saeed Tavazoie at Princeton University, New Jersey, and his colleagues have demonstrated that bacteria can learn and exhibit anticipatory behaviour (I. Tagkopoulos et al. Science 320, 1313-1317; 2008). They show computationally and experimentally that Escherichia coli can learn temporal correlations between environmental stimuli - for example, that an increase in temperature is followed by a decrease in oxygen levels allowing the bacteria to predict and prepare for future environmental changes.

The researchers show that this associative learning is accomplished by rewiring of biochemical networks. Strikingly, they also show that, like many of us, E. coli quickly 'unlearn' (in fewer than 100 generations) what they had learned in a new situation.

Now we know that bacteria can be taught such tricks, it will be interesting to see if we can use novel combinations of environmental stimuli to train microbes to efficiently convert biomass into energy sources, such as hydrogen or butanol. By providing E. coli with such an educational stimulus package, we may be able to boost the global economy.

Discuss this paper at http://blogs. nature.com/nature/journalclub 